\title{
DRAG FORCE OF A POROUS PARTICLE MOVING AXISYMMETRICALLY IN A CLOSED CAVITY OF MICROPOLAR FLUID
}

\author{
Krishna Prasad Madasu \\ Department of Mathematics, National Institute of Technology, Raipur-492010 \\ Chhatisgarh, India \\ madaspra.maths@nitrr.ac.in,kpm973@gmail.com
}

Received: 13 April 2019; Accepted: 13 January 2020

\begin{abstract}
The present paper deals with the problem of an incompressible axisymmetric creeping flow caused by a porous spherical particle in a spherical cavity filled with micropolar fluid. Depending on the kind of cell model, appropriate boundary conditions are used on the surface of sphere and spherical cavity. Drag force on the porous particle in the presence of a cavity is calculated to determine the correction factor to the Stokes law. A general expression for the hydrodynamic force acting on the porous sphere and, hence, for the wall correction factor of the sphere are obtained. The special cases of the porous sphere in viscous fluid, zero permeability solid sphere in micropolar fluid and viscous fluid are obtained in open and closed cavity respectively.
\end{abstract}

MSC 2010: 76A05, 76D07,76S05

Keywords: micropolar fluid, Stokes flow, sphere, concentric, drag force

\section{Introduction}

The study of the creeping flow past spherical particles with a porous layer has been an active field of research as it plays a crucial role in diverse applications, such as flow of blood through the lungs and human tissues, dealing with drainage and irrigation, oil and gas production from reservoirs and biological applications etc. Practically, particles are in assemblage being solid or porous in nature. The unit cell model technique is used to investigate the effect of inter particle interaction on transport properties. Problems of the hydrodynamic interactions using the cell model have been treated extensively in the past. One can find useful knowledge in this area in Happel and Brenner [1]. Considerable work has been reported on viscous fluid past a porous particles [2-5]. Srinivasacharya [6] studied flow past a porous approximate spherical shell. Dassios et al. [7] examined the Stokes flow problem using cell models for a spheroidal particle. Faltas and Saad [8] investigated Stokes flow past an assemblage of slip eccentric spherical particle-in-cell models. 
In 1966, Eringen $[9,10]$ introduced the theory of micropolar fluids to describe the motion of fluids with microstructure. A micropolar fluid is a physically relevant model that has many applications such as extrusion of polymer fluids, solidification of liquid crystals, animal blood and engineering applications. Extensive reviews about the applications of micropolar fluids can be found in Ariman et al. [11] and in a book by Lukaszewicz [12]. Rao and Rao [13] studied the Stokes flow of micropolar fluid past a rigid sphere. Ramkissoon and Majumdar [14] investigated the Stokes flow of a micropolar fluid past an axi-symmetric body and concluded that the drag in the classical fluid is less than that of the micropolar fluid. Using the spin boundary condition, Hoffmann et al. [15] evaluated the hydrodynamic force exerted on a solid sphere moving with a constant velocity in a micropolar fluid. Saad [16] analyzed the Stokes axisymmetrical translational motion of a spheroidal particle in an unbounded micropolar fluid and the motion of a spheroidal particle at the instant it passes the centre of a spherical envelope filled with a micropolar fluid. Sherief et al. [17] investigated the axisymmetric rectilinear and rotary oscillations of a spheroidal particle in an incompressible micropolar fluid using slip boundary condition. Sherief et al. [18] discussed Stokes flow of a micropolar fluid past an assemblage of spheroidal particle-in-cell models with slip. Saad [19] has adopted mathematical and numerical techniques to investigate the creeping flow of an incompressible micropolar fluid past an assemblage of a slip eccentric spherical particle in bounded medium. Sherief et al. [20] investigated the axisymmetric motion of a solid spherical particle in a micropolar fluid within a non-concentric spherical cavity. Sherief et al. [21] studied the steady translational and rotational motion of two rigid spheres with slip surfaces immersed in an incompressible micropolar fluid. Iyengar and Radhika [22] investigated the problem of flow of an incompressible micropolar fluid past a porous prolate spheroidal shell with a solid core region kept in an infinite expanse of the fluid. Mishra and Gupta [23] studied creeping motion of a permeable sphere in a spherical container filled with a micropolar fluid.

Recently, the interaction problem between particles and an envelope of spherical shape containing micropolar fluid has attracted the attention of a small number of authors. Motivated by the above-mentioned works. To the author's knowledge, the drag force exerted on the porous sphere in a closed cavity filled with micropolar fluid has not been studied yet. We have used the Stokesian approximation for the micropolar fluid that governs in the cavity region and Darcy's law for the porous region. As boundary conditions, continuity of the normal velocity, pressure, and slip boundary condition for tangential stresses and zero microrotation across the interface are employed. Different boundary conditions are suggested for the cell surface which takes into account the effect of surrounding particles on the particle in the center of the cell (Happel [24], Kuwabara [25]). The stream function (and thus the velocity) components and pressure (both for the flow inside and outside) are calculated. The flow examined is axially symmetric in nature. The drag force and wall correction factor exerted on the porous sphere in a spherical cavity is studied numerically and presented graphically. 


\section{Mathematical modelling}

The present mathematical model investigates the unit cell model for the steady axisymmetric flow of an incompressible micropolar fluid of viscosities $\left(\mu, \kappa, \alpha_{0}, \beta_{0}, \gamma_{0}\right)$ past a porous sphere of radius $a$ in a spherical cavity of radius $b(a<b)$. All the porous spherical particles are homogeneously and randomly distributed in an incompressible micropolar fluid. The porous medium is assumed to be homogeneous and isotropic. The particle being at rest while the spherical cavity moves in the negative $z$-direction with constant velocity $U$. The external and internal regions are denoted by I and II, respectively (Fig. 1). Neglecting the inertial and gyro-inertial terms in the field equations by assuming very small Reynold's numbers for the micropolar fluid i.e., Stokesian approximation is assumed. The equations of motion for the region outside porous sphere are the equations governing steady flow of an incompressible micropolar fluid with the absence of body force and body couple are given by Eringen $[9,10]$.

$$
\begin{gathered}
\nabla \cdot \vec{q}^{(1)}=0, \\
\nabla p^{(1)}+(\mu+\kappa) \nabla \times \nabla \times \vec{q}^{(1)}-\kappa \nabla \times \vec{v}=0, \\
\kappa \nabla \times \vec{q}^{(1)}-2 \kappa \vec{v}-\gamma_{0} \nabla \times \nabla \times \vec{v}+\left(\alpha_{0}+\beta_{0}+\gamma_{0}\right) \nabla \nabla \cdot \vec{v}=0,
\end{gathered}
$$

where $\vec{q}^{(1)}, v$, and $p^{(1)}$ are the velocity vector, microrotation vector, and fluid pressure at any point, respectively. $\mu$ is the viscosity coefficient of the classical viscous fluid and $\kappa$ is the vortex viscosity coefficient. The remaining constants $\alpha_{0}, \beta_{0}$, and $\gamma_{0}$ are the gyroviscosity coefficients.

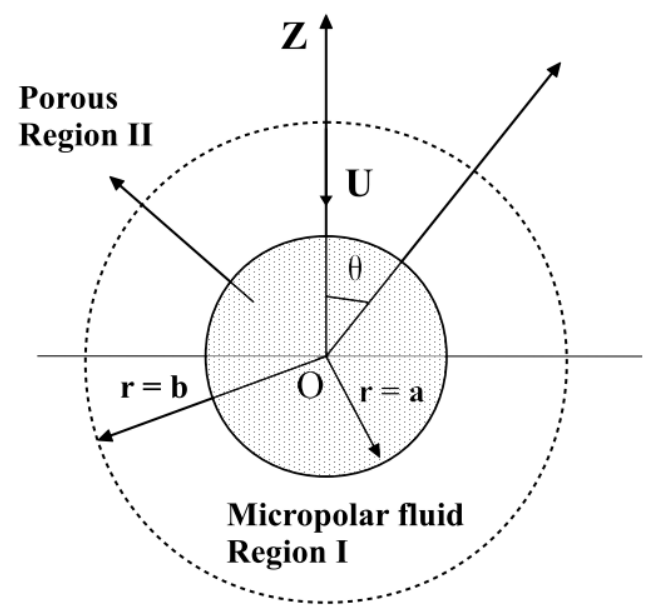

Fig. 1. Geometry of the problem 
For internal region, equations of motion are

$$
\begin{gathered}
\nabla \cdot \vec{q}^{(2)}=0, \\
\vec{q}^{(2)}=-\frac{k}{\mu} \nabla p^{(2)},
\end{gathered}
$$

where $\vec{q}^{(2)}$ is the velocity, $p^{(2)}$ is the pressure.

Let the origin of the spherical coordinate system $(r, \theta, \varphi)$ be fixed at the centre of the porous sphere and $\left(\vec{e}_{r}, \vec{e}_{\theta}, \vec{e}_{\varphi}\right)$ be the unit of base vectors. Since the flow of the fluid is in the meridian plane and the flow is axially symmetric, all the physical quantities are independent of $\varphi$. Hence, we assume the velocity vectors and microrotation vector as

$$
\begin{gathered}
\vec{q}^{(i)}=q_{r}^{(i)}(r, \theta) \vec{e}_{r}+q_{\theta}^{(i)}(r, \theta) \vec{e}_{\theta}, i=1,2, \\
\vec{v}=v_{\varphi}(r, \theta) \vec{e}_{\varphi} .
\end{gathered}
$$

\section{Solution of the problem}

In view of the incompressibility condition $\nabla \cdot \vec{q}^{(i)}=0, i=1,2$, we introduce the stream functions $\psi^{(i)}(r, \theta), i=1,2$ through

$$
q_{r}^{(i)}=\frac{1}{r^{2}} \frac{\partial \psi^{(i)}}{\partial \xi}, q_{\theta}^{(i)}=\frac{1}{r \sqrt{1-\xi^{2}}} \frac{\partial \psi^{(i)}}{\partial r}, i=1,2 \text { and } \xi=\cos \theta
$$

Introducing the following non-dimensional variables $r=a \tilde{r}, \quad \psi^{(i)}=U a^{2} \tilde{\psi}^{(i)}$, $p^{(i)}=\frac{\mu U}{a} \tilde{p}^{(i)}$, and $v_{\varphi}=\frac{U}{a} \tilde{v}_{\varphi}$. Eliminating pressure from (1) and (2) and dropping tildes, we get the following dimensionless equations:

$$
E^{4}\left(E^{2}-\lambda^{2}\right) \psi^{(1)}=0,
$$

with the microrotation vector as

$$
\begin{gathered}
v_{\varphi}=\frac{1}{2 r \sqrt{1-\xi^{2}}}\left(E^{2} \psi^{(1)}+\frac{(2+\tau)}{\tau \lambda^{2}} E^{4} \psi^{(1)}\right), \\
E^{2} \psi^{(2)}=0,
\end{gathered}
$$


where

$E^{2}$ denotes the Stokes stream function operator given by $E^{2}=\frac{\partial^{2}}{\partial r^{2}}+\frac{\left(1-\xi^{2}\right)}{r^{2}} \frac{\partial^{2}}{\partial \xi^{2}}, \lambda^{2}=\frac{a^{2} \kappa(2+\tau)}{\gamma_{0}(1+\tau)}, \tau=\frac{\kappa}{\mu}$.

Following Happel and Brenner [1], the solution of (6) which is regular at infinity is

$$
\psi^{(1)}=\left[A r^{2}+B r^{-1}+C r^{4}+D r+E \sqrt{r} K_{3 / 2}(\lambda r)+F \sqrt{r} I_{3 / 2}(\lambda r)\right] \vartheta_{2}(\xi)
$$

substituting this in (7), we get the microrotation component as

$v_{\varphi}=\frac{1}{r \sqrt{1-\xi^{2}}}\left[5 C r^{2}-D r^{-1}+\lambda^{2} \frac{(1+\tau)}{\tau} E \sqrt{r} K_{3 / 2}(\lambda r)+\lambda^{2} \frac{(1+\tau)}{\tau} F \sqrt{r} I_{3 / 2}(\lambda r)\right] \vartheta_{2}(\xi)$.

Where $I_{3 / 2}(\lambda r)$ and $K_{3 / 2}(\lambda r)$ are the modified Bessel function of the first kind and second kind of order $\frac{3}{2}$, respectively. $\vartheta_{2}(\xi)$ is the Gegenbauer function of the first kind of order 2 and degree $-\frac{1}{2}$.

The solution of (8) which is finite as $r \rightarrow 0$ is

$$
\psi^{(2)}=G r^{2} \vartheta_{2}(\xi),
$$

The boundary conditions which are physically realistic and mathematically consistent for the present problem, at the surface of porous sphere $r=1$, are:

(i) Continuity of the normal velocity components:

$$
\left(\vec{q}^{(1)}-\vec{q}^{(2)}\right) \cdot \vec{n}=0,
$$

(ii) Continuity of pressures:

$$
p^{(1)}=p^{(2)},
$$

(iii) Generalised Beavers-Joseph slip boundary condition [3]:

$$
\left(\vec{n} \cdot t^{(1)}\right) \cdot \vec{s}=\alpha \sigma\left(\vec{q}^{(1)}-\vec{q}^{(2)}\right) \cdot \vec{s}
$$

(iv) No-slip condition of microrotation:

$$
v_{\varphi}=0
$$

Where $\vec{n}$ and $\vec{s}$ are the normal unit and tangential unit vectors at the porous sphere surface pointing into the fluid. $\sigma$ is a dimensionless slip coefficient and its value depends on the internal structure of the porous surface. $t^{(1)}$ is the stress tensor in the micropolar fluid. $\alpha^{2}=\frac{a^{2}}{k}$ is the permeability parameter. 
The boundary conditions on the outer cell surface $r=\frac{1}{\eta}\left(\eta=\frac{a}{b}\right)$ are Happel and Kuwabara models. In the two models, it is assumed that

(v) Radial velocity on the cell surface is continuous:

$$
q_{r}^{(1)}=-U \cos \theta,
$$

(vi) Vanishing of microrotation component as follows:

$$
v_{\varphi}=0
$$

A third boundary condition used, respectively, in each of the mentioned models is as follows:

(vii) The Happel model [24] assumes that the tangential stress vanishes on the cell surface

$$
t_{r \theta}^{(1)}=0
$$

(viii) The Kuwabara model [25] assumed that the vorticity vanishes on the cell surface i.e., the flow is assumed to be potential

$$
\operatorname{curlq}^{(1)}=0 \text {. }
$$

\section{Drag on the body}

The drag force acting on the porous sphere in a spherical cavity can be evaluated by using the formula

$$
\begin{gathered}
F=\left.2 \pi a^{2} \int_{0}^{\pi} r^{2}\left(t_{r r}^{(1)} \cos \theta-t_{r \theta}^{(1)} \sin \theta\right)\right|_{r=1} \sin \theta d \theta \\
F=2 \pi a U \mu(2+\tau) D
\end{gathered}
$$

where $D$ is a constant whose expression is lengthy and therefore not presented here.

$$
F_{\infty}=2 \pi a U \mu(2+\tau) \Delta_{1}
$$

where:

$$
\begin{aligned}
& \Delta_{1}=-6 K_{3 / 2}(\lambda) \lambda^{2} \alpha^{2}(1+\tau) w \Delta, \\
& \Delta^{-1}=\lambda\left(-2 K_{1 / 2}(\lambda) \alpha^{2} \tau w+K_{3 / 2}(\lambda) \lambda(1+\tau)\left(4 \alpha^{3} \sigma+3\left(2 \alpha^{2}+w\right)(2+\tau)\right)\right), \\
& w=(2+\alpha \sigma+\tau) .
\end{aligned}
$$


The normalised drag force (wall correction factor $W_{c}$ ) is defined as the ratio of the drag force experienced by the porous sphere in the closed cavity to the drag force exerted on the porous sphere in an infinite expanse of fluid. With the aid of Eq.'s. (21) and (22), this becomes

$$
W=\frac{F}{F_{\infty}}
$$

\subsection{Special cases}

(i) Non-Newtonian fluid (Micropolar fluid) past a solid sphere

If $\alpha \rightarrow \infty$ and $\eta \rightarrow 0$ in Eq. (22), drag on a solid sphere in the case of uniform streaming in an unbounded micropolar fluid yields

$$
F=-6 \pi a U \mu\left[\frac{(2+\tau)(1+\tau)(1+\lambda)}{2 \lambda(1+\tau)+2+\tau}\right]
$$

which agrees with the result obtained by Rao and Rao [13] and Ramkissoon and Majumdar [14].

(ii) Viscous fluid past a solid sphere

When $\tau \rightarrow 0$ and $\gamma_{0} \rightarrow 0$ in Eq. (24), the drag force reduces to the classical result of Newtonian fluid as

$$
F=-6 \pi a U \mu
$$

(iii) Viscous fluid past a porous sphere using Darcy's law

When $\tau \rightarrow 0$ and $\gamma_{0} \rightarrow 0$ in Eq. (22), the drag reduces to

$$
F=-4 \pi a U \mu\left[\frac{3 \alpha^{2}(2+\alpha \sigma)}{6+6 \alpha^{2}+3 \alpha \sigma+2 \alpha^{3} \sigma}\right]
$$

which agrees with the result obtained by Jones [4], Davis and Stone [5], and Srinivasacharya [6].

(iv) Viscous fluid past a porous sphere using Darcy's law in cell models When $\tau \rightarrow 0$ and $\gamma_{0} \rightarrow 0$ in Eq. (21), the particle behaves like a porous sphere in a spherical cell. In this case, the drag force reduces to

$$
\begin{gathered}
F_{H p}=-4 \pi a U \mu\left[\frac{\alpha^{2}\left(\alpha \sigma\left(2 \eta^{5}+3\right)-6\left(\eta^{5}-1\right)\right)}{\Delta_{2}}\right] \\
F_{K u}=-4 \pi a U \mu\left[\frac{15 \alpha^{2}(2+\alpha \sigma)}{\Delta_{3}}\right]
\end{gathered}
$$


Where:

$\Delta_{2}=6\left(\eta^{5}-1\right)-6 \alpha^{2}\left(\eta^{6}-\eta^{5}-\eta+1\right)+\alpha^{3} \sigma\left(2 \eta^{6}-3 \eta^{5}+3 \eta-2\right)-3 \alpha \sigma\left(4 \eta^{5}+1\right)$
$\Delta_{3}=-30\left(2 \eta^{3}+1\right)-6 \alpha^{2}\left(\eta^{6}-6 \eta+5\right)+2 \alpha^{3} \sigma\left(\eta^{6}-5 \eta^{3}+9 \eta-5\right)-15 \alpha \sigma\left(2 \eta^{3}+1\right)$

If $\alpha \rightarrow \infty(k=0)$ in Eq. (27) and (28), the porous sphere become a solid sphere.

The drag force reduces to the following expressions:

$$
\begin{aligned}
& F_{H p}=-4 \pi a U \mu\left[\frac{2 \eta^{5}+3}{2 \eta^{6}-3 \eta^{5}+3 \eta-2}\right] \\
& F_{K u}=-30 \pi a U \mu\left[\frac{1}{\eta^{6}-5 \eta^{3}+9 \eta-5}\right]
\end{aligned}
$$

\section{Numerical results}

The wall correction factor $W_{c}$ acting on the porous sphere in presence of the spherical cavity is numerically computed for different values of the micropolarity parameter $\tau$, volume fraction of the spherical particle $\delta=\eta^{3}(0<\delta<1)$, slip coefficient $\sigma$ for all the four models. In all numerical computation of the wall correction factor, we assumed the value of $\frac{\gamma_{0}}{\mu a^{2}}=0.3$. The results are shown in Figure 2.

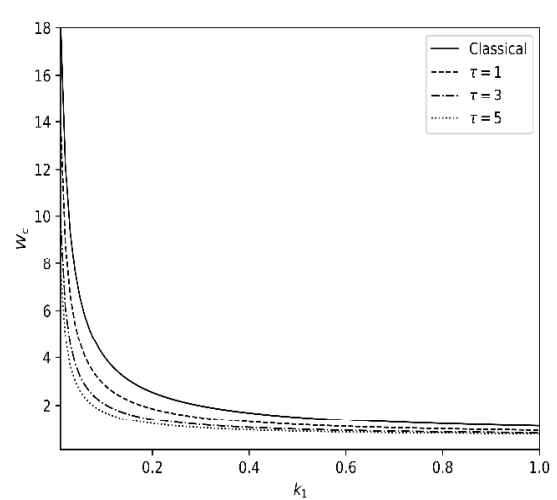

(a) Happel model

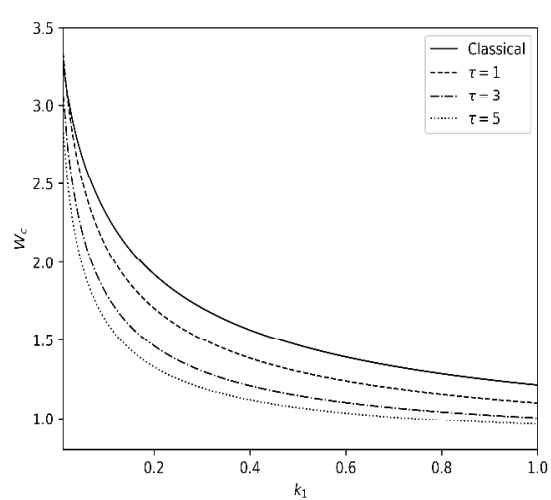

(b) Kuwabara model

Fig. 2. Variation of the wall correction factor $W_{c}$ versus permeability $k_{1}$ for different values of the micropolarity parameter $\tau$, volume fraction $\delta=0.6$, and slip coefficient $\sigma=1.5$ are fixed

Figure 2 illustrates the variation of the wall correction factor $W_{c}$ versus permeability $k_{1}\left(=\frac{1}{\alpha}=\frac{\sqrt{k}}{a}\right)$ for the two models with the volume fraction $\delta$ and slip coefficient $\sigma$ as fixed, for various values of the micropolarity parameter $\tau$. The curve 
with $\tau=0$ represents the result for a porous sphere in a spherical cell filled with viscous fluid. Whereas the curve with non-zero $\tau$ denotes the result for a porous sphere in a spherical cell containing micropolar fluid. For any specified finite value of $\tau$, the wall correction factor $W_{c}$ decreases monotonically with an increase in permeability $k_{1}$. Also, it can perceived from the figure that there is a decrease in the wall correction factor compared to the case when both of the fluids are Newtonian. Thus, we conclude that the wall correction factor of a micropolar fluid is smaller than a classical fluid.

Table 1 . Wall correction factor $W_{c}$ for different values of the permeability $k_{1}$, volume fraction $\delta=0.6$, and slip parameter $\sigma=1.5$

\begin{tabular}{|c|c|c|c|c|}
\hline \multicolumn{5}{|c|}{$W_{c}$} \\
\hline$k_{1}$ & $\tau \rightarrow 0$ & $\tau=1$ & $\tau=3$ & $\tau=5$ \\
\hline \multicolumn{5}{|c|}{ Happel model } \\
\hline 0.01 & 18.43452 & 14.10618 & 9.912145 & 7.8337751 \\
\hline 0.1 & 4.045744 & 2.864520 & 2.061655 & 1.713590 \\
\hline 0.3 & 1.975506 & 1.481843 & 1.164650 & 1.032753 \\
\hline 0.5 & 1.494154 & 1.168727 & 0.966130 & 0.884735 \\
\hline 0.7 & 1.276584 & 1.028901 & 0.878885 & 0.820504 \\
\hline 0.9 & 1.152288 & 0.949764 & 0.830173 & 0.785013 \\
\hline \multicolumn{5}{|c|}{ Kuwabara model } \\
\hline 0.01 & 20.55833 & 14.646545 & 9.930382 & 7.725818 \\
\hline 0.1 & 3.571170 & 2.514980 & 1.775670 & 1.444677 \\
\hline 0.3 & 1.607056 & 1.211781 & 0.933850 & 0.809820 \\
\hline 0.5 & 1.173077 & 0.925426 & 0.750674 & 0.672971 \\
\hline 0.7 & 0.979529 & 0.797938 & 0.669567 & 0.612665 \\
\hline 0.9 & 0.869314 & 0.725434 & 0.623623 & 0.578612 \\
\hline
\end{tabular}

Table 2. Wall correction factor $W_{c}$ for different values of the volume fraction $\delta$, micropolarity parameter $\tau \rightarrow 0$, gyroviscosity coefficient $\gamma_{0} \rightarrow 0$ and slip parameter $\sigma \rightarrow \infty$

\begin{tabular}{|c|c|c|c|c|}
\hline \multicolumn{5}{|c|}{$W_{c}$} \\
\hline$\delta$ & $k_{1} \rightarrow 0$ & $k_{1}=0.1$ & $k_{1}=0.3$ & $k_{1}=0.5$ \\
\hline \multicolumn{5}{|c|}{ Happel model } \\
\hline 0.01 & 1.476622 & 1.389806 & 1.285555 & 1.225163 \\
\hline 0.1 & 3.110796 & 2.385492 & 1.805009 & 1.556165 \\
\hline 0.3 & 10.134775 & 3.70535 & 1.976162 & 1.512364 \\
\hline 0.5 & 37.907321 & 3.751566 & 1.672713 & 1.226201 \\
\hline 0.7 & 230.435118 & 3.229821 & 1.368581 & 0.992668 \\
\hline 0.9 & 7992.544916 & 2.74371 & 1.153383 & 0.835242 \\
\hline \multicolumn{5}{|c|}{ Kuwabara model } \\
\hline 0.01 & 1.607247 & 1.483523 & 1.341125 & 1.26155 \\
\hline 0.1 & 3.80932 & 2.598787 & 1.806822 & 1.505358 \\
\hline 0.3 & 12.983524 & 3.627523 & 1.819275 & 1.370377 \\
\hline 0.5 & 46.862429 & 3.578775 & 1.573797 & 1.150303 \\
\hline 0.7 & 265.055099 & 3.161315 & 1.337919 & 0.970189 \\
\hline 0.9 & 8395.466081 & 2.73732 & 1.150685 & 0.833286 \\
\hline
\end{tabular}


Table 1 shows the numerical results of wall correction factor $W_{c}$ for different values of permeability parameter $k_{1}$. It is observed that $W_{c}$ is a monotonic decreasing function of permeability $k_{1}$ for the two cell models. As micropolarity parameter $\tau$ increases, $W_{c}$ decreases. The results for the case of the impermeable solid sphere in a cell model are presented in the Table 2. The results are in good agreement compared to the values obtained by Faltas and Saad [8].

\section{Conclusions}

In this paper, the steady axisymmetric Stokes flow of an incompressible micropolar fluid past a porous sphere located in a concentric spherical particle-in-cell models have been investigated theoretically. In the Stokes flow regime, the Darcy's law and Stokes equations for the fluid flow field applicable to these axisymmetric motions are analytically solved. The hydrodynamic drag force exerted by the micropolar fluid on the porous particle-in-cell models is a function of the permeability parameter, slip coefficient, micropolarity parameter, separation parameter. It is found that the wall correction factor of a porous sphere-in-cell model filled with micropolar fluid is smaller than that of a porous sphere-in-cell model filled with viscous fluid. For given values of volume fraction $\delta$ and slip parameter $\sigma$, the correction factor decreases with an increase in the permeability $k_{1}$ and micropolarity parameter $\tau$. There is a slight decrease in correction factor with an increase in slip coefficient $\sigma$.

\section{Acknowledgements}

The author (M. Krishna Prasad) acknowledges and wishes to thank CCOST, Raipur (C.G), India, for carrying out this research work under grant no.: 2543/CCOST/MRP/2016.

\section{References}

[1] Happel, J., \& Brenner, H. (1965). Low Reynolds Number Hydrodynamics. N.J.: Prentice-Hall, Englewood Cliffs.

[2] Joseph, D.D., \& Tao, L.N. (1964). The effect of permeability in the slow motion of a porous sphere in a viscous liquid. Journal of Applied Mathematics and Mechanics, 44, 361-364.

[3] Beavers, G.S., \& Joseph, D.D. (1967). Boundary conditions at a naturally permeable wall. Journal of Fluid Mechanics, 30, 197-207.

[4] Jones, I.P. (1973). Low Reynolds number flow past a porous spherical shell. Mathematical Proceedings of the Cambridge Philosophical Society, 73, 231-238.

[5] Davis, R.H., \& Stone, A.H. (1993). Flow through beds of porous particles. Chemical Engineering Science, 48(23), 3993-4005.

[6] Srinivasacharya, D. (2007). Flow past a porous approximate spherical shell. Journal of Applied Mathematics and Physics, 58, 646-658. 
[7] Dassios, G., Hadjinicolaou, M., Coutelieris, F.A., \& Payatakes, A.C. (1995). Stokes flow in spheroidal particle-in-cell models with Happel Kuwabara boundary conditions. International Journal of Engineering Science, 33, 1465-1490.

[8] Faltas, M.S., \& Saad, E.I. (2011). Stokes flow past an assemblage of slip eccentric spherical particle-in-cell models. Mathematical Methods in the Applied Sciences, 34, 1594-1605.

[9] Eringen, A.C. (1966). Theory of micropolar fluids. Journal of Mathematics and Mechanics, 16, $1-18$.

[10] Eringen, A.C. (2001). Microcontinuum Field Theories II. New York: Springer, Fluent media.

[11] Ariman, T., Turk, M.A., \& Sylvester, N.D. (1974). Applications of microcontinuum fluid mechanics. International Journal of Engineering Science, 12, 273-293.

[12] Lukaszewicz, G. (1999). Micropolar fluids-theory and applications. Basel: Birkhauser.

[13] Rao, S.K.L., Rao, P.B. (1970). The slow stationary flow of a micropolar liquid past a sphere. Journal of Engineering Mathematics, 4, 209-217.

[14] Ramkissoon, H., Majumdar, S.R. (1976). Drag on an axially symmetric body in the Stokes flow of micropolar fluid. Physics of Fluids, 19, 16-21.

[15] Hoffmann, K.H., Marx, D., \& Botkin, N.D. (2007). Drag on spheres in micropolar fluids with non-zero boundary conditions for microrotations. Journal of Fluid Mechanics, 590, 319-330.

[16] Saad, E.I. (2008). Motion of a spheroidal particle in a micropolar fluid contained in a spherical envelope. Canadian Journal of Physics, 86, 1039-1056.

[17] Sherief, H.H., Faltas, M.S., \& Saad, E.I.(2013). Slip at the surface of an oscillating spheroidal particle in a micropolar fluid. ANZIAM Journal, 55, E1-E50.

[18] Sherief, H.H., Faltas, M.S., Ashmawy, E.A., \& Nashwan, M.G. (2015). Stokes flow of a micropolar fluid past an assemblage of spheroidal particle-in-cell models with slip. Physica Scripta, 90(5), 055203.

[19] Saad, E.I.(2014). Motion of a slip sphere in a nonconcentric fictitious spherical envelope of micropolar fluid. ANZIAM J., 55, 383-401.

[20] Sherief, H.H., Faltas, M.S., \& El-Sapa, S. (2019) Axisymmetric creeping motion caused by a spherical particle in a micropolar fluid within a nonconcentric spherical cavity. European Journal of Mechanics-B Fluids, 77, 211-220.

[21] Sherief, H.H., Faltas, M.S., \& El-Sapa, S. (2019). Interaction between two rigid spheres moving in a micropolar fluid with slip surfaces. Journal of Molecular Liquids, 290, 111165.

[22] Iyengar, T.K.V., \& Radhika, T. (2011). Stokes flow of an incompressible micropolar fluid past a porous spheroidal shell. Bulletin of the Polish Academy of Sciences: Technical Sciences, 59, 63-74.

[23] Mishra, V., \& Gupta, B.R. (2017). Motion of a permeable shell in a spherical container filled with non-Newtonian fluid. Applied Mathematics and Mechanics, 38(12), 1697-1708.

[24] Happel, J. (1958). Viscous flow in multiparticle systems, Slow motion of fluids relative to beds of spherical particles. AIChE Journal, 4, 197-201.

[25] Kuwabara, S. (1959). The forces experienced by randomly distributed parallel circular cylinders or spheres in a viscous flow at small Reynolds numbers. Journal of the Physical Society of Japan, 14, 527-532. 